\title{
Projeto piloto de informatização dos prontuários do setor de motilidade ocular extrínseca da Santa Casa de São Paulo
}

\author{
Pilot project of computerization of charts of the extrinsic ocular motility \\ sector of the Santa Casa of São Paulo
}

\author{
Patrícia Novita Garcia ${ }^{1}$ \\ Mauro Goldchmit ${ }^{2}$ \\ Carlos Ramos de Souza-Dias ${ }^{3}$
}

\footnotetext{
Trabalho realizado no Departamento de Oftalmologia da Santa Casa de Misericórdia de São Paulo - São Paulo (SP) - Brasil

Fellow do Setor de Córnea e Doenças externas do Departamento de Oftalmologia da Santa Casa de Misericórdia de São Paulo e Chefe do Setor de Ultra-sonografia Ocular do Departamento de Oftalmologia da Santa Casa de Misericórdia de São Paulo - São Paulo (SP) - Brasil.

Doutor; Chefe do Setor de Motilidade Extrínseca Ocular do Departamento de Oftalmologia da Faculdade de Ciências Médicas da Santa Casa de Misericórdia São Paulo - São Paulo (SP) - Brasil.

Professor Titular da Faculdade de Ciências Médicas da Santa Casa de Misericórdia de São Paulo - São Paulo (SP) - Brasil.

Endereço para correspondência: Patrícia Novita Garcia. Rua Minas Gerais, 408 - Apto. 62 - São Paulo (SP) CEP 01244-010

E-mail: patricianovita@yahoo.com.br

Recebido para publicação em 23.09.2007

Última versão recebida em 24.03.2008

Aprovação em 30.04.2008
}

\begin{tabular}{|c|}
\hline RESUMO \\
\hline $\begin{array}{l}\text { Objetivo: Desenvolver um sistema de apoio à pesquisa no campo da } \\
\text { informática, baseado na busca automática de informações sobre a porcen- } \\
\text { tagem de pacientes com determinadas características e o cruzamento dessas } \\
\text { informações entre si. Métodos: Utilizou-se um computador com pro- } \\
\text { cessador Pentium III } 650 \mathrm{MHz}, 128 \mathrm{MB} \text { de RAM, placa de vídeo de } 32 \mathrm{MB} \text {, } \\
20 \text { MB livres em disco rígido e capacitado com Windows } 98 / 2000 / \mathrm{XP} \text {. O } \\
\text { banco de dados utilizado para armazenar as informações é o Interbase } \\
\text { versão } 6.1 \text { e o programa foi desenvolvido em linguagem Delphi } 5.0 \text {. Foram } \\
\text { cadastradas } 304 \text { fichas dos pacientes operados de esotropia no setor de } \\
\text { Motilidade Ocular Extrínseca do Departamento de Oftalmologia da Fa- } \\
\text { culdade de Ciências Médicas da Santa Casa de Misericórdia de São Paulo, } \\
\text { no período de } 02 / 07 / 76 \text { a 09/03/92. Para cadastrar os pacientes no pro- } \\
\text { grama, foi elaborado um questionário, com as possíveis variações clínicas } \\
\text { relevantes para esse tipo de desvio. Foram apresentados exemplos de } \\
\text { totalizações e cruzamentos de informações. Resultados: Esse novo progra- } \\
\text { ma poderá contribuir para a pesquisa científica, agilizando o levantamento } \\
\text { de dados. Após o cadastro dos pacientes, qualquer tipo de levantamento, } \\
\text { seleção de um grupo específico de pacientes ou cruzamento de dados } \\
\text { poderá ser obtido em segundos. Conclusão: Pode ser feito em todas as } \\
\text { especialidades oftalmológicas, variando o questionário de acordo com } \\
\text { cada especialidade. Assim um relatório atual poderá ser acessado instanta- } \\
\text { neamente quando se deseja fazer uma pesquisa ou consulta. }\end{array}$ \\
\hline
\end{tabular}

Descritores: Projeto de sistemas; Sistemas computadorizados de registros médicos; Sistemas de informação; Transtornos da motilidade ocular; Oftalmologia

\section{INTRODUÇÃO}

A aplicação da informática em saúde tem-se difundido com sucesso em todo o mundo, para apoio tanto ao processo de tomada de decisão, quanto ao aprendizado $^{(1)}$. Com computadores inteligentes, desenvolver-se-iam programas capazes de armazenar e processar vasta quantidade de conhecimentos, assim como organizar-se-iam bancos de dados, formando, desse modo, o conceito de inteligência artificial ${ }^{(2)}$, que pode ser entendida como o conjunto de técnicas e métodos de programação usados para tentar resolver problemas de forma mais eficiente ${ }^{(3-4)}$.

Em vários países europeus, a informática em saúde é uma realidade; na área médica, difundida em suas universidades ${ }^{(5-7)}$. 
Toda essa evolução na área de informática voltada à medicina ocorre por ser o aprendizado dificultado pela grande quantidade de informações que, na área da saúde, aumenta a uma velocidade nunca antes imaginada, e pela dificuldade no levantamento de dados para realização da pesquisa científica ${ }^{(8-11)}$.

Muitos esforços já foram feitos na tentativa de melhorar o atendimento na área da saúde. O uso de modernas ferramentas da informática e das telecomunicações são recursos promissores. Com a utilização delas pelo médico, o paciente passa a ser acompanhado de maneira rápida, organizada e eficaz ${ }^{(12-14)}$.

O objetivo deste trabalho foi desenvolver um sistema de apoio à pesquisa no campo da informática, baseado na busca automática de informações sobre o número de pacientes com determinadas características e o cruzamento dessas informações entre si, para a obtenção de um banco de dados atualizado de pacientes da Seção de Motilidade Extrinseca Ocular do Departamento de Oftalmologia da Santa Casa de Misericórdia de São Paulo.

\section{MÉTODOS}

Para o desenvolvimento deste trabalho, utilizou-se computador com processador Pentium III $650 \mathrm{MHz}, 128 \mathrm{MB}$ de RAM, placa de vídeo de $32 \mathrm{MB}, 20 \mathrm{MB}$ livres em disco rígido e capacitado com Windows 98/2000/XP, não funciona com outros sistemas operacionais. $\mathrm{O}$ banco de dados utilizado para armazenar as informações é o Interbase versão 6.1 e o programa foi desenvolvido em linguagem Delphi 5.0 por Alexandre Manzo Nubile (analista de sistemas). O programa pode funcionar em rede, mas ainda não pode ser acessado pela internet. $\mathrm{O}$ programa segue o cronograma abaixo:

Após preenchimento dos campos "Usuário" e "Senha", a página apresenta duas opções: "Cadastro" e "Relatórios". O "Cadastro" tem um ícone "Pesquisa" que deve ser iniciado.

No ícone "Pesquisa" são solicitados o título (definido como ESTRABISMO), local onde foi realizada a pesquisa (SANTA CASA), questionário (sexo, idade, diagnóstico, refração, acuidade visual, valor do desvio na posição primaria do olhar, ambliopia, quantas vezes operou, quem operou, etc.) e possíveis respostas (Figura 1).

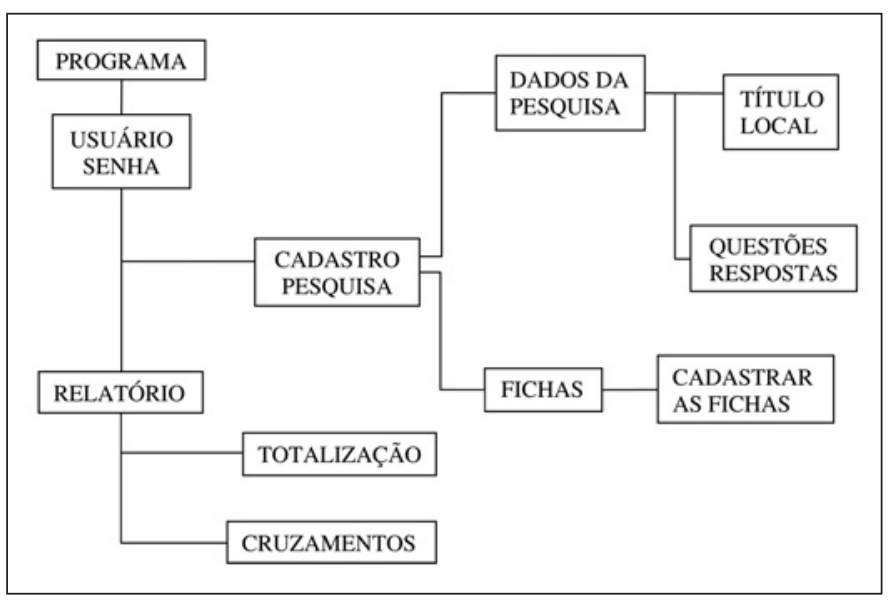

É permitido o cadastro de até 9.999 perguntas, algumas delas apresenta duas ou mais variáveis, como a acuidade visual, que se define como do olho direito e esquerdo, devem ser colocadas separadas como duas questões e esse cadastro pode ser ampliado se necessário.

Estando todos os dados da pesquisa já com as perguntas e as respostas relacionadas, inicia-se o cadastro das fichas (Figura 2).

Durante o cadastramento das fichas, pode-se acrescentar uma questão que seja relevante para a pesquisa, bem como possíveis respostas.

Após o cadastramento dos dados, procede-se à verificação dos "Relatórios"; pode-se optar pelas "Totalizações" ou pelos "Cruzamentos". Nas “Totalizações", as questões aparecem para ser escolhidas (Figura 3).

Desse modo, são fornecidos os totais, com seus respectivos valores em porcentagem. Pode-se optar por selecionar todas as questões ao mesmo tempo ou selecionar somente algumas. Caso precise-se saber os números das fichas, os registros, com determinada resposta, basta solicitar "listar números das fichas", selecionar a resposta desejada e "imprimir".

Ao selecionar os "Cruzamentos" dentro do ícone "Relatórios", uma nova janela aparece (Figura 4).

Assim, aparecem as questões, com suas respectivas respostas, que devem ser selecionadas, de modo que se separem as respostas que se desejem cruzar. Por exemplo: deseja-se saber quais são os pacientes do sexo feminino, com 3 anos de idade e com acuidade visual conta dedos (Cd) a 2 metros. Cruzam-se essas informações e obtém-se a porcentagem dos pacientes com acuidade visual $\mathrm{Cd} 2 \mathrm{~m}$, dentre os do sexo feminino, com 3 anos e amblíopes. Podem-se selecionar quaisquer dados com quaisquer outros para obter-se a porcentagem de um em relação ao outro.

Com as porcentagens já exibidas, pode-se solicitar o número dessas fichas ao selecionar-se "Listar número das fichas"; desse modo obtém-se o registro das fichas cruzadas.

Com finalidade didática e para melhorar o entendimento em relação ao funcionamento e utilidade do programa, foram cadastradas 304 fichas dos pacientes operados de esotropia, no setor de Motilidade Ocular Extrínseca do Departamento de Oftalmologia da Faculdade de Ciências Médicas da Santa Casa de Misericórdia de São Paulo, no período de 2 de julho de 1976 a 9 de março de 1992. Para cadastrar os pacientes no programa, foi elaborado um questionário, com as possíveis variações clínicas relevantes nesse tipo de desvio. Com as questões e as fichas já cadastradas, as totalizações de todas as perguntas ocorrem em 0,4 segundo. Esse tempo pode variar dependendo do número de questões totalizadas e do número de fichas cadastradas.

\section{DISCUSSÃO}

Os sistemas de banco de dados para pesquisa não apenas congregam especialistas em computação das mais diversas áreas, mas também se apresentam como um tema já bem estabelecido em informática médica, exercendo grande fascínio em todos os que, de uma forma ou de outra, já ouviram falar deles. 


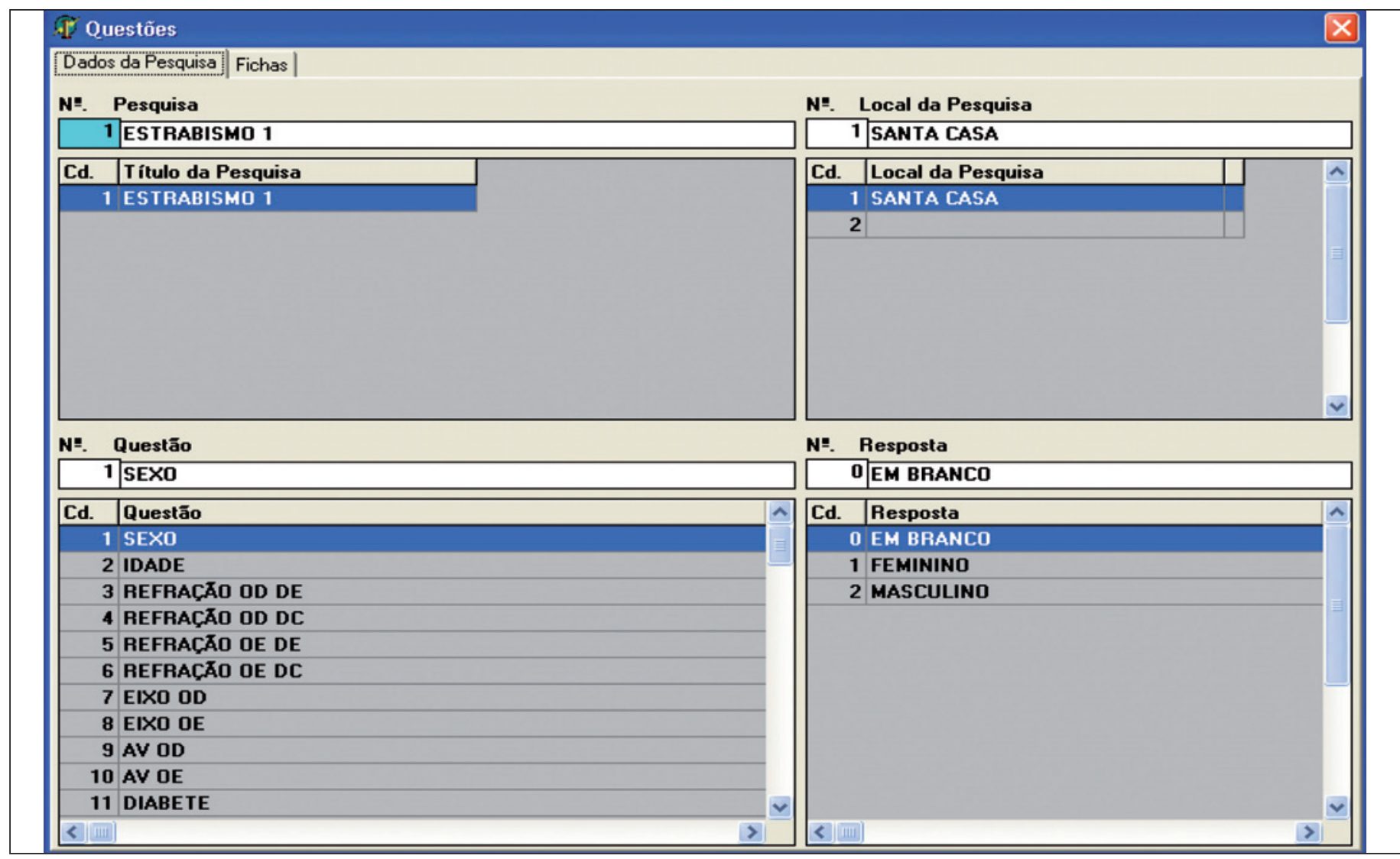

Figura 1 - Dados da pesquisa, questionário e respostas. Projeto de informática em pesquisa oftalmológica/SANTA CASA/SP-2004.

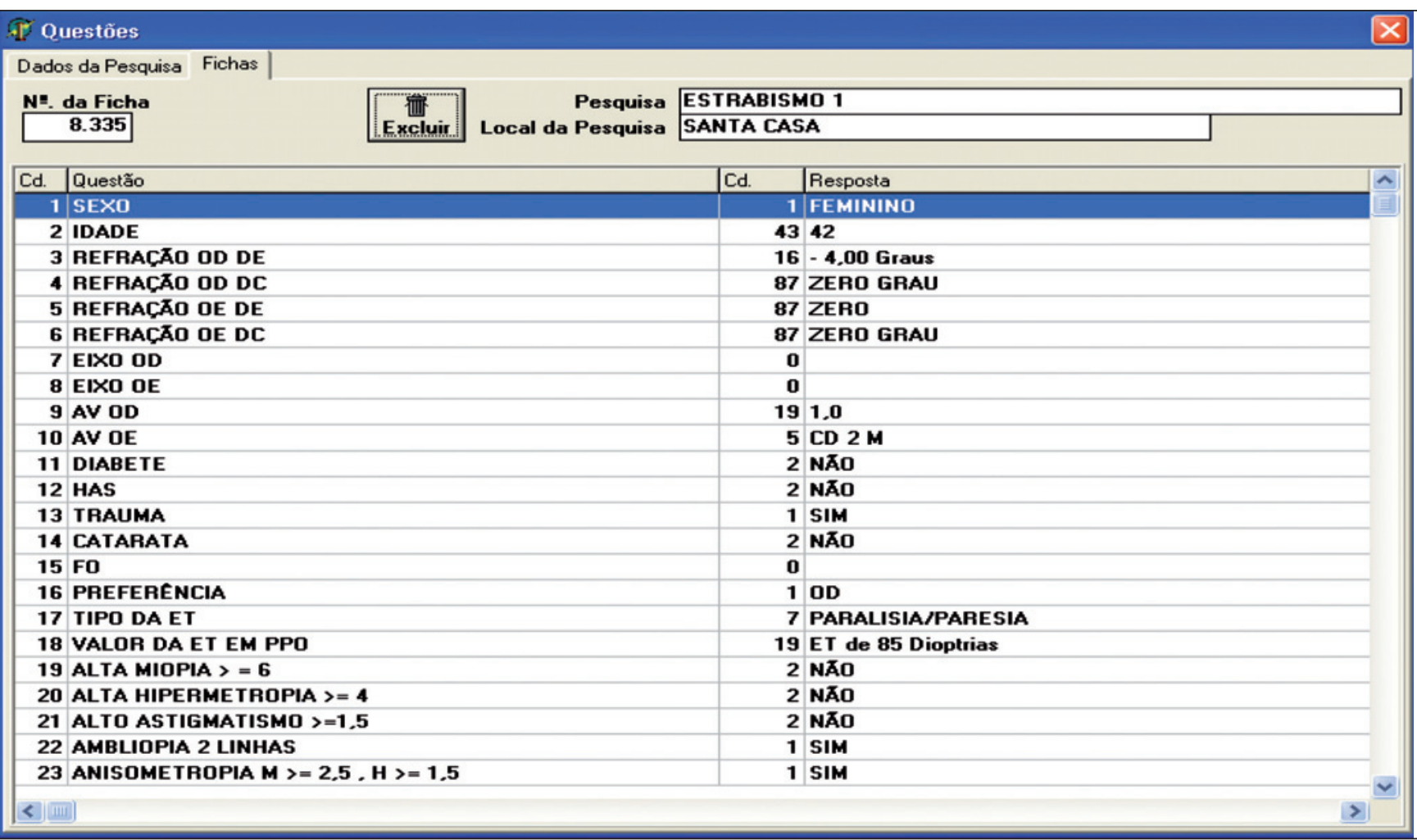

Figura 2 - Fichas para serem cadastradas. Projeto de informática em pesquisa oftalmológica/SANTA CASA/SP-2004. 


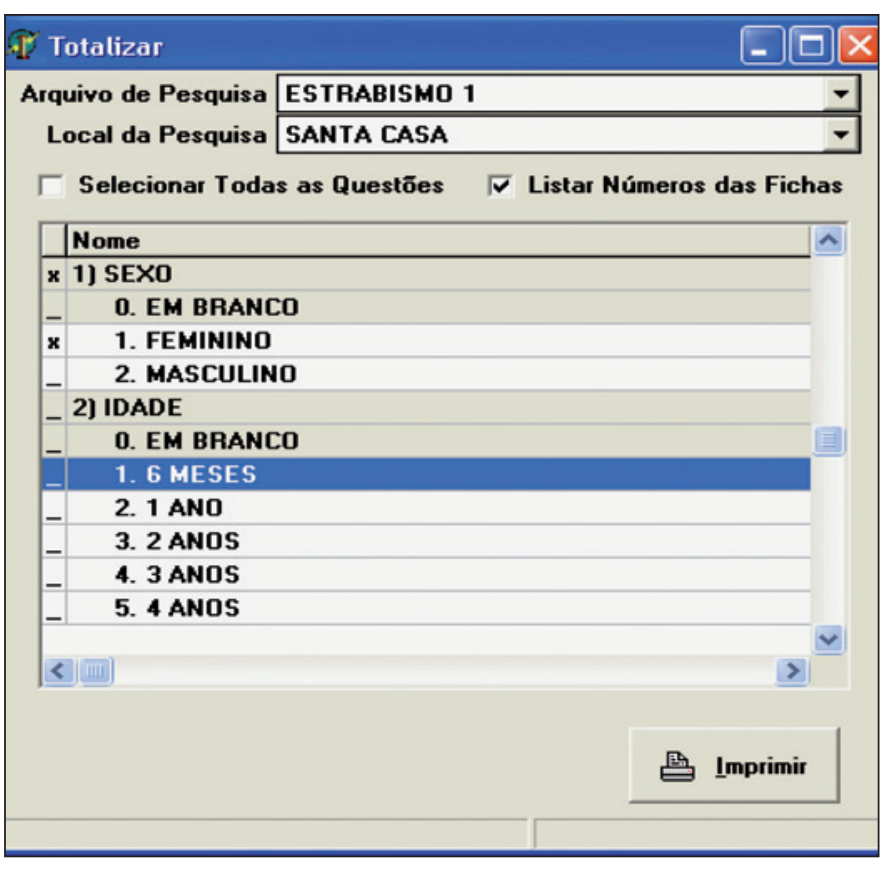

Figura 3 - Totalizações. Projeto de informática em pesquisa oftalmológica/SANTA CASA/SP-2004.
Dentro desse espírito, e sabendo-se das limitações existentes no acesso tradicional dos profissionais da saúde ao levantamento de dados para realização de pesquisa científica sobre determinados temas, a informática impõe-se como instrumento de eficácia incomparável. De forma bastante patente, os bancos de dados apresentam facetas de versatilidade que jamais poderiam ser encontradas em um livro.

Se a questão é cruzar certo número de informações com determinada finalidade e tal tarefa é muito demorada para o pesquisador, está perfeitamente adequada para um banco de dados ou bases de conhecimento. Qual o profissional que não gostaria de poder digitar os sinais e sintomas que deseja selecionar entre os seus pacientes e obter sem demora um elenco de todos esses dados?

O apoio dos recursos de informática possibilita enorme salto qualitativo e quantitativo na pesquisa científica. Os resultados evidentes e as informações relacionadas aos pacientes são adquiridas de forma rápida, clara e não geram dúvidas.

Com a utilização do "software" de pesquisa, pode-se traçar um perfil de atendimento ao paciente e detectar os principais diagnósticos e dados clínicos desejados, facilitando o levantamento e a organização dos dados.

\begin{tabular}{|c|c|c|c|c|c|}
\hline \multicolumn{6}{|c|}{ Cruzamentos } \\
\hline \multirow{2}{*}{\multicolumn{2}{|c|}{$\begin{array}{r}\text { Arquivo de Pesquisa } \\
\text { Local da Pesquisa }\end{array}$}} & \multirow{2}{*}{ 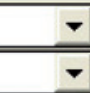 } & \multirow[t]{2}{*}{ Г Listar Números das Fichas } & \multirow[t]{2}{*}{ 咀 } & \multirow[t]{2}{*}{ D } \\
\hline & & & & & \\
\hline & Questōes & $\overline{\widehat{A}}$ & \multicolumn{2}{|l|}{ Questōes } & \multirow[t]{2}{*}{ 스 } \\
\hline 6 & \multicolumn{2}{|l|}{ 1) SEXO } & \multicolumn{2}{|l|}{ 2) IDADE } & \\
\hline 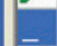 & \multicolumn{2}{|l|}{ 2) IDADE } & \multicolumn{2}{|l|}{ 3) REFRAÇĀO OD DE } & \\
\hline - & \multicolumn{2}{|l|}{ 3) REFRAÇAO OD DE } & \multicolumn{2}{|l|}{ 4) REFRAÇAO OD DC } & \\
\hline- & \multicolumn{2}{|l|}{ 4) REFRAÇAOO OD DC } & \multicolumn{3}{|l|}{ 5) REFRAÇAO OE DE } \\
\hline- & \multicolumn{2}{|l|}{ 5) REFRACXAO OE DE } & \multicolumn{3}{|l|}{ 6) REFRAÇĀO OE DC } \\
\hline - & \multicolumn{2}{|l|}{ 6) REFRAÇĀO OE DC } & \multicolumn{2}{|l|}{ 7) EIXO OD } & 国 \\
\hline- & \multicolumn{2}{|l|}{ 7) EIXO OD } & \multicolumn{2}{|l|}{ 8) EIXO OE } & \\
\hline - & \multicolumn{2}{|l|}{ 8) EIXO OE } & \multicolumn{2}{|l|}{ 9) AV OD } & \\
\hline - & \multicolumn{2}{|l|}{ 9) AV OD } & \multicolumn{3}{|l|}{ 10) AV OE } \\
\hline _ & \multicolumn{2}{|l|}{ 10) AV OE } & \multicolumn{3}{|l|}{ 11) DIABETE } \\
\hline - & \multicolumn{2}{|l|}{ 11) DIABETE } & \multicolumn{3}{|l|}{ 12) HAS } \\
\hline - & 12) HAS & $\checkmark$ & _ 13) TRAUMA & & $\checkmark$ \\
\hline$<$ & IIII) & $>$ & $<$ (iii) & $>$ & \\
\hline Qu & estōes Selecionadas & $\hat{\wedge}$ & Questōes Selecionadas & & $\widehat{A}$ \\
\hline & SEXO & & 2) IDADE & & \\
\hline & 0. EM BRANCO & & O. EM BRANCO & & \\
\hline & 1. FEMININO & & 1. 6 MESES & & \\
\hline & 2. MASCULINO & & 2. 1 ANO & & \\
\hline & & & 3. 2 ANOS & & \\
\hline & & & 4. 3 ANOS & & \\
\hline & & & 5. 4 ANOS & & \\
\hline & & & 6. 5 ANOS & & \\
\hline & & & 7. 6 ANOS & & \\
\hline & & $v$ & & & $\checkmark$ \\
\hline$<$ & IiiI) & $>$ & < IIII) & $>$ & \\
\hline
\end{tabular}

Figura 4 - Cruzamentos. Projeto de informática em pesquisa oftalmológica/SANTA CASA/SP-2004. 
Assim, a implantação do sistema de banco de dados para gerenciamento de informações clínicas de pacientes trouxe os seguintes benefícios:

1) Agilizou e organizou as tarefas de levantamento de dados para a pesquisa científica;

2) Propiciou análises estatísticas percentuais, um dos fatores preponderantes no traçado do perfil de atendimento;

3) Construiu uma base de dados para armazenar as informações criptografadas dos pacientes (sexo, idade, acuidade visual e dados do exame oftalmológico), coletadas durante a consulta clínica;

4) Recuperou os registros clínicos de forma rápida, pela estruturação adequada dos arquivos de dados, em que os eventos são todos direcionados ao paciente.

\section{CONCLUSÃO}

Em nosso serviço, esse novo "software" poderá contribuir para a pesquisa científica, agilizando o levantamento de dados. Após o cadastro dos pacientes, qualquer tipo de levantamento, seleção de um grupo específico de pacientes ou cruzamento de dados poderá ser obtido em segundos. O programa desenvolvido poderá ser aplicado a todas as subespecialidades da oftalmologia, variando o questionário de acordo com as características de cada uma. Assim um relatório atual pode ser acessado instantaneamente quando se deseje fazer uma pesquisa ou consulta.

\section{ABSTRACT}

Purpose: To develop a system of support to the research in the field of the computer science, based on the automatic search of information, on the percentage of patients with certain characteristics and the crossing of this information among themselves. Methods: A computer with Pentium III $650 \mathrm{MHz}$ processor, $128 \mathrm{MB}$ RAM, $32 \mathrm{MB}$ video plate, free $20 \mathrm{MB}$ in the hard disk and fitted with Windows 98/2000/XP. The data base used to store the information is Interbase version 6.1 and the program was developed in Delphi 5.0 language. Three hundred and four charts of esotropia-operated patients were registered in the Extrinsic Ocular Motility Sector of the Departament of Ophthalmology of the College of Medical Sciences of the Santa Casa of Misericórdia of São Paulo, in the period from 07/02/1976 to 03/09/1992. To include the patients in the program, a questionnaire was elaborated, with the relevant clinical variations of this type of misalignment. Examples of totalizations and crossings of information were presented. Results: This new program will be able to contribute to scientific research, accelerating data collection. After registering the patients any type of survey, election of a specific group of patients or crossing of data may be obtained in seconds. Conclusion: This can be made for all ophthalmologic specialties, varying the questionnaire in accordance with each specialty.

Keywords: System design; Medical records systems, computerized; Information systems; Ocular motility disorders; Ophthalmology

\section{REFERÊNCIAS}

1. Bellazzi R, MontanI S, Riva A, Stefanelli M. Web-based telemedicine systems for home-care: technical issues and experiences. Comput Methods Programs Biomed. 2001;64(3):175-87.

2. Barnard CM, Goldyne ME. Evaluation of an asynchronous teleconsultation system for diagnosis of skin cancer and other skin diseases. Telemed J E Health. 2000;6(4):379-84

3. Mantas JD. Nursing informatics- generic high level trainning in informatics for nurse. General applications for learning and Education (NIGHTINGALE). In: I European Conference on Health Telematic Education- HTE’96. Proceedings. Corfu Island, Greece: sept. 1996.

4. Sabbatini RM. Bancos de dados em medicina. São Paulo: Instituto Brasileiro de Informação em Saúde e editora; 1988.

5. Fernandes AP. Sistema especialista difuso de apoio ao aprendizado do traumatismo dento-alveolar utilizando recursos multimídia [tese] Florianópolis: Universidade Federal de Santa Catarina; 1997.

6. Sabbatini RM. O ensino da informática aplicada à Medicina. Roteiros e bibliografia básica. Rev Informédica. 1994;2(8):5-12.

7. Bronzino JD. The biomedical engineering handbook. Boca Raton, Florida: CRC Press; 1995.

8. Sabbatini RM. An improved undergraduate curriculum for teaching medical informatics to medical and nursing students. In: Van Bemmel JH, Zvárová J, editors. Knowledge, information and medical education. Amsterdam: North Holland; 1991. p.67-78.

9. Fernandes AP. Aided learning expert system to diagnosis and treatment of dental traumatism in odontology pratical clinical class. In: I European Conference about Health Telematic Education - HTE’96. Proceedings. Corfu Island, Greece; Sept. 1996.

10. Sabbatini RM. Automação de arquivos médicos em microcomputadores. In Rodrigues RJ, editor. A informática e o administrador hospitalar. São Paulo: Pioneira; 1987. p.37-9.

11. Palombo CR, Maccari Filho M, El-Guindy MM, Sabbatini RM. Diagface: um banco de conhecimentos e sistema especialista para o diagnóstico de patologias orofaciais. In: IV Congresso Brasileiro de Informática em Saúde. Sociedade Brasileira de Informática em Saúde. Anais. Porto Alegre; 1994.

12. Wada M, Suzuki R, Murakami T. Super-high-definition image systems for telemedicine. J Telemed Telecare. 2000;6 Suppl 2:S85.

13. Aarnio P, Jaatinen P, Hakkari K, Halin N. A new method for surgical consultations with videoconference. Ann Chirur Gynaecol. 2000;89(4):336-40.

14. Davenport JC, Hammond P. The acquisition and validation of removable partial denture design knowledge. J Oral Rehabil. 1996;23(3):152-7. 